\title{
Comunicación Interauricular Tipo Ostium Secundum Análisis y Seguimiento de 80 Casos
}

\author{
Dr. Ignacio Hernández N.1-2; Dra. Odette Farrú A.1; Dr. Gastón Duffau 0.1
}

\author{
Ostium Secundum Atrial Septal Defect in Children
}

\begin{abstract}
The clinical and hemodynamic data in 80 children with Ostium Secundum atrial septal defect (OS-ASD) were analyzed. Sixty one of them were followed after surgical repair during a period of one month to six years. It must be emphasized that OS-ASD rarely causes significant symptoms in childhood and that it is infrecuently diagnosed in the first $y$ ears of life. Nevertheiess the clinical features of OS-ASD are typical allowing an appropriate diagnosis and even an estimate of the magnitude of the shunt. ln contrast with the experience in adults the surgical treatnent of chiddren with OS-ASD is safe and with low motbidity $(0 \%$ mortality and 4,9\%morbidity in our serie). For that reason it is very important to make an early diagnosis and surgcal repair.
\end{abstract}

La Comunicación Interauricular tipo Ostium Secundum (CIA-OS) constituye alrededor del $15 \%$ de las cardiopatías congénitas en el niño, siendo después de la Comunicación interventricular la más frecuente. El defecto permite un cortocircuito de izquierda a derecha entre las auriculas cuya dirección y magnitud dependen del tamaño del defecto y de la resistencia al flujo sanguíneo hacia los respectivos ventrículos; normalmente el ventrículo izquierdo crea una mayor resistencia al llenado diastólico desde la aurícula que el ventrículo derecho, más delgado y distensible. Es así como en la CIA-OS el flujo pulmonar es 2 a 3 veces mayor que el sistémico siendo esto tolerado durante años sin gran dificultad. Hacia la cuarta década de la vida, sin embargo, se complica frecuentemente con hipertensión pulmonar, trastornos del ritmo a insuficiencia cardíaca, determinando con ello una significativa menor sobrevida $1 \cdot 2 \cdot 3 \cdot 4 \cdot 5 \cdot 6 \cdot 7$.

La corrección quirúrgica de la CIA $-O S$ es efectiva y con baja morbimortalidad si se realiza en niños. De ahí la importancia de diagnóstico y tratamiento precoces, aún en sujetos asintomáticos.

El objeto de esta revisión fue mostrar la forma de presentación, evolución natural y post-operatoria de la CIA-OS en nuestro medio.

Por constituir embriológica, clínica y hemodinámicamente una entidad diferente optarmos por excluir las Comunicaciones Interauriculares tipo Ostium Primum.

\section{MATERIAL Y METODO}

De 156 fichas clínicas de pacientes portadores de CIA-OS controlados en la Unidad de Cardio-

1 Unidad de Cardiología, Hospital Roberto del Río.

2 Trabajo de incorporación a la Sociedad Chilena de Pediatría. logía del Hospital Roberto del Río se seleccionaron 80 por contener información completa. En ellas se efectuó un análisis retrospectivo clínico, auscultatorio-fonocardiográfico y hemodinámico. Los niveles de significación estadística se realizaron según la distribución de Students.

El seguimiento clínico de los vî̀ios operados fluctuó entre 1 mes y 6 años. Todos los pacientes están vivos.

\section{RESULTADOS}

La distribución por sexo mostró una relación mujer/hombre de 1,5:1. La edad promedio de hallazgo de soplo fue de 5 años y medio, describiéndose éste, a pesar de no ser característico, en dos recién nacidos en quienes posteriormente se demostró una CIA-OS.

El análisis del estado nutricional considerando peso y talla según tablas nacionales, demostró déficit de peso en $37 \%$ de los casos, siendo de grado I en $82 \%$ de ellos. Compromiso de la talla se encontró en 13,7\%. Al buscar asociaciones entre la magnitud del cortocircuito y el déficit de peso, se encontró en los niños desnutridos (peso/ edad) una relación entre los flujos sistémica $y$ pulmonar (Qp: Qs) promedio de 2,5: 1 y en los eutróficos de $2,45: 1$; cifras prácticamente iguales.

Los sintomas estaban ausentes en $58,8 \%$ de los niños, había disnea de esfuerzo en $30 \%$, bronquitis recidivante en $6,2 \%$ e insuficiencia cardíaca congestiva en $5 \%$ ( 4 casos, todos menores de 18 meses). E1 Qp: Qs promedio de los niños asintomáticos fue $2,3: 1$ y el de sintomáti$\cos 2,78: 1$; cifras estadisticamente significativas $(\mathrm{t}=2.217 ; \mathrm{p}<0.05)$.

En todos los casos con excepción de los menores de 1 año 6 meses, se encontró segundo ruido desdoblado fijo. La intensidad del soplo fue de $3 / 6$, como promedio; ubicándose entre el 
segundo y tercer espacio intercostal izquierdo en la región paraesternal. Había soplo diastólico con carácter de rodada tricuspídea en $90 \%$ de los casos; al relacionarla con el grado de cortocircuito, los niños con rodada tenian Qp: Qs promedio de 2,61: 1 y sin-ella 2,21: 1 , lo que carece de significado estadístico (t: $1.508 ; \mathrm{p}>0.05$ ).

En los electrocardiogramas (ECG) habia bloqueo incompleto de rama derecha (BIRD) en 74 $(92,5 \%)$ de los pacientes, hipertrofia ventricular derecha (HVD) en 42 (52,5\%), T negativa aislada en $22(27,5 \%)$, bloqueo aurículo-ventricular de primer grado en $5(6,3 \%)$ y ritmo coronario en 5 $(6,3 \%)$. El EEG era normal en 6 ninios $(7,5 \%)$ con Qp: Qs promedio 1,79: 1.

En el estudio hemodinámico efectuado en 70 de los 80 pacientes el Qp: Qs promedio fue de 2,53\%: 1. En 4 (5,7\%) se obtuvo Qp: Qs menor de $1,5: 1$, en $25(35,7 \%)$ de 1,5 a 2,1 y en 41 $(58,6 \%)$ mayor de $2,1: 1$. La presión promedio del ventrículo derecho fue de $34,1 \mathrm{mmHg}$., en la atteria pulmonar de $27,3 \mathrm{mmHg}$. y en el capilat pulmonar de $7,3 \mathrm{mmHg}$.

De los 80 niños se intervinieron quirúrgica. mente 61 (76\%): 32 en el Hospital Clínico de la Universidad de Chile, 23 en el Hospital Clinico de la Universidad Católica y 6 en el Hospital Luis Calvo Mackenna. La edad promedio de operación fue de 9 años. Al relacionar la magnitud del cortocircuito con el tamaño diámetro mayor del defecto en los 20 niños en quienes se registoó la información quirúrgica durante la operación, se encontró en las ClA-OS con Qp: Qs menor de 2: 1 un tamaño promedio de $22,9 \mathrm{~mm}$; $35 \mathrm{~mm}$. cuando Qp: Qs estaba entre 2,1: 1 y 3: 1 y $38,6 \mathrm{~mm}$. en aquellas con Qp: Qs sobre 3,1: 1 .

No hubo mortalidad quirúrgica. El seguimiento de los pacientes operados fluctuó entre 1 mes y. 6 años (promedio 18 meses). La morbilidad post-operatoria $(4,9 \%)$ estuvo dada por 2 casos de Fibrilación auricular, uno de aparición inmediata después de la intervención y otro 5 meses despuess, asociada a Enfermedad del Nódulo. Estos 2 niños se han controlado satisfactoriamente con tratamiento médico. E1 resto de los pacientes operados están asintomáticos y su examen clínico es normal.

\section{comentarios}

La CIA-OS es una cardiopatía frecuente en los niños. Entre los pacientes controlados en nuestra Unidad corresponde al $12,1 \%$ de los casos. En la literatura las cifras dadas oscilan entre 7 y $17 \%^{8-9-10}$, siguiendo siempre a la Comunicación Interventricular, que es el defecto más frecuente. Una incidencia mayor de CIA-OS en mujeres concuerda con lo clásicamente descrito $^{1}-7 \cdot 9-9$ - 10 . Es una cardiopatía de diagnóstico tardio, hecho corroborado en nuestros niños. La edad de hallazgo del soplo, que generalmente coincide con la primera consulta al cardiólogo, fue de 5 años y medio. En 2 niños se describió un soplo desde el período de recién nacido, sin ser típico de CIA-OS. La auscultación se va haciendo característica hacia $\operatorname{los} 2$ años de edad, lo que se explica por el aumento del cortocircuito de izquierda a derecha que ocurre a medida que disminuye la hipertrofia del ven. trículo derecho propia del recién nacido y del lactante $e^{9 \cdot 11 \cdot 12 \cdot 13}$.

En relación con la tardia pesquisa, la mayoría de los portadores de ClA-OS son asintomáticos. La disnea de esfuerzo resulta un sintoma dificil de valorar en los nirios, especialmente si es leve; en todos los casos estudiados fue de poca magnitud. Los problemas broncopulmonares secundarios fueron escasos. La insuficiencia cardiaca congestiva es rara en la CIA-OS y cuando se manifiesta ocurre fundamentalmente en lactantes $^{9} \cdot 11 \cdot 12 \cdot 13 \cdot 14 \cdot 15 \cdot 16 \cdot 17 \cdot 18 \mathrm{y}$ en adultos mayores de $40^{1} \cdot 2 \cdot 4 \cdot 7 \cdot 19$. La insuficiencia cardíaca del lactante con ClA-OS no tiene una clara explicación fisiopatológica ya que su defecto no difiere con los encontrados en nixios mayores sin insuficiencia, atribuyéndose a modificaciones precoces de la circulación pulmonar $y$ de la capacitancia del ventriculo derecho ${ }^{12}$ - $13 \cdot 14 \cdot 20$. En nuestros casos, los 4 níios (5\%) con insuficiencia cardíaca eran lactantes menores de 18 meses.

En el examen físico se encuentra frecuentemente rodada tricuspídea. Su existencia se relaciona directamente con la magnitud del cottocircuito del que constituye un buen indicador ${ }^{20}$.

En la mayoría de los pacientes con CIA-OS existe BIRD y un número también importante tienen HVD; ambos, junto con la historia y el examen físico, permiten una buena aproximación al diagnóstico. Respecto del ECG, se describe en la CIA-OS la presencia de onda $T$ negativa en V3, V4 ó V5 como un elemento frecuente ${ }^{21}$ y $\mathrm{P}-\mathrm{R}$ prolongado ${ }^{8} \cdot 9 \cdot 10 \cdot 22$, en proporciones similares a las nuestras. Otro hecho electrocardiográfico interesante es la presencia de ritmo coronario en 5 de los casos analizados; 4 antes y 1 después de la intervención. Los 5 eran CIA-OS del tipo Cava Superior. Se piensa que la vecindad del defecto con el nơdulo sinusal, compromete a éste anátomofuncionalmente, dando lugar a ubicación inferior del marcapasos ${ }^{10} \cdot 23 \cdot 24 \cdot 25$. Estos 5 casos se asociaban con drenaje venoso parcial anómalo, hecho descrito en la literatura como frecuente ${ }^{23}$. En consideración a lo anterior se advierte que la presencia de ritmo coronario es 
un elemento sugerente de CIA-OS tipo Cava Superior.

La CIA-OS es una cardiopatía con cortocircuito moderado, lo que se comprueba en nuestros casos. Esto explica en alguna medida la pobreza de los sintomas y lo tardío del diagnostico. En ningún caso se demostró hipertensión pulmonar, apoyando la baja incidencia descrita de esta complicación en los nifíos, en contraste con el adulto, que en proporción cercana a $50 \%$ tiene hipertensión pulmonar después de los 40 años $1 \cdot 2 \cdot 4 \cdot 7$.

La Radiologia es concordante con CIA-OS en prácticamente todos los casos, en especial si son sintomáticos. Dado lo subjetivo de su interpretación y la demostración, en una revisión previa, de falta de correlación entre radiología y grado de cortocircuito $^{20}$, en esta oportunidad no se ha insistido en ella.

En nuestros pacientes no encontramos casos de cierre espontáneo de CIA-OS como se ha descrito en otras publicaciones $26-27 \cdot 28 \cdot 29$. $30-31$

En virtud de lo típico de las manifestaciones clinicas, el aporte del electrocardiograma y las radiografias ha sido posible prescindir del estudio hemodinámico en un número importante de niños, enviándoles a operar sin sondeo cardíaco. La ecocardiografía ha permitido notables aportes minimizando la necesidad de emplear técnicas invasivas.

La morbimortalidad quirúrgica es escasa en nuestra serie, contrastando con lo que sucede cuando la intervención se realiza en adultos, en quienes los trastornos del ritmo y los derivados de la hipertensión pulmonar son frecuentes, determinando mayor morbilidad y mortalidad $\mathbf{1}$ - 4 . $5-32-33$

\section{RESUMEN}

Se exponen los hallazgos clínicos y hemodinámicos en 80 niños portadores de Comunicación Interauricular tipo Ostium Secundum (CIA-OS). Se efectúa un seguimiento post-. operatorio en 61 de ellos, durante un periodo de tiempo que fluctuó entre 1 mes y 6 años.

Se concluye que la ClA-OS es una cardiopatía poco sintomática en la infancia cuyo diagnóstico infrecuentemente se realiza en los primeros afros de la vida. Sin embargo existen elementos clínicos característicos para diagnosticarla que incluso permiten predecir el grado de shunt.

Contrariamente con lo que sucede en el adulto, el tratamiento quirúrgico en niros con CIA-OS es de bajo riesgo y de baja mortalidad (\% de mortalidad y $4,9 \%$ de morbilidad en nuestra serie). De ahi la importancia de diagnóstico y tratamiento quirúrgico precoces.

\section{REFERENCIAS}

' Craig R.J., Selzer A.: Natural history and prognosis of atrial septal defect. Circulation 37: 805, 1968.

${ }^{2}$ Crawford $W \cdot A$ : A reapprasal of life expectancy with atrial shunts of the secundum type. Diseases of the chest $48: 357,1965$.

3 Dalen J.E., Haynes F. W., Dexter L.: Life expectancy with atrial septal defect. Influence of complicating pulmonary vaseular disease. J.A.M.A. 200: 112, 1967.

4 Daven K.S., Pakrashi B.C., Wooler A., Ionescu M.I. Atrial septal defect in adults. Clinical and hemodinamic results of surgery. Amer. J. Cardiol, 31: 7, 1973.

5 Durán G., Reyes J., Perales L. Quijada P., Allamand $F$. Comunicación Interauricular. Tratamiento quirürgico en adultos. Arch. Soc. Cit. Chile 24: 255. 1972.

${ }^{6}$ Meruane J, D'Acuña V., Carranza C. Comunicación Interautricular en Antofagasta. Rev. Med. del Norte. EnetolJunio: 152, 1970.

3 Zaver A., Nadas A.: Atrial septal defect secundum type. Circulation 32: 24, 1965.

${ }^{B}$ Dupuis $C$. Kachoner J., Pernot C., Quero-Jimenez $M$, Rey C.: Cardiologie Pediatrique. Flammarion Medicine Sciences, Cap. 15, 1981.

${ }^{9}$ Keith J.D., Rowe R.D., Vlad P.: Heart disease in infancy and childhood. 2nd Ed. Chap. 22. Macmillan Publishing $\mathrm{Co}$, New York, Toronto and London, 1981.

10 Perloff $J . K$.: The clinical recognition of congenital heart disease. 2nd Ed. Chap. 15 W.B. Saunders, Philadelphia, London and Toronto. 1978.

11 Nakamura F.L., Hanck A.J., Nadas A.S.: Atrial septal defect in infants Pediatrics 34: 101, 1964.

12 Dimich $f$., Steinfeid L., Park S.C.: Symptomatic atrial septal defect in infants. Amer. Heart J. 85 : $601,1973$.

13 Farrú O., Braga M.: Comunicación Interauricular ipo Ostium Secundum con insuficiencia cardíaca en el lactante. Pediatría (Santiago) 23: 64, 1980.

14 Hastreiter A., Wennmark J., Miler R., Patu M. Secundum atrial septal defects with congestive heart failure during infancy and early childhood. Am. Hcart J. 64: 467, 1962.

15 Hunt C.E. Lucas R.V.: Symptomatic atrial septal defect in infancy. Circulation 47: 1042, 1973.

16 Kavaragh-Gray D.: atrial septal defect in infancy. Canad. Med. Ass. J. 89: 491, 1963.

17 Pagtakhon R.D., Hartmann A.F., Goldring D. Kiscane $J$.: The valve incompetent foramen oval. A repori of 7 infants with left to tight atrial shunt. J. Pediatr. 71 : 848, 1967.

18 Weinberg M., Miller A., Hastreiter A.R., Raffens. perger J.G., Fell E.H., Buchelers H.G.: Congestive heart failure in clildren with atrial septal defect. $J$. Thot. Cardiov, Surg. 5 : 81, 1966.

19 Nasralleh A.T., Hall R.J., Garcia E., Leachman R.D., Cooley D.A.: Surgical repair of atrial septal defect in patients over 60 years age. Circulation 53: 329 , 1976.

20 Rees A., Forrù O., Rodriguez R.: Phonocardiographic, radiological and haemodinamic correlation in atrial septal defect. B. Heart 34: 781, 1972.

21 Duffau G., Ahumado J.: Estuäio de algunos aspectos de la repolarización ventricular en la C.I.A. Pedjatría $11: 183,1968$. 
22 Levin A.R., Haft J, Engle M.M., Ehlers K.H., Klein A.A.: Intracardiac conduction intervals in children with congenital heart disease. Circulation 55: 286, 1977.

${ }^{3} 3$ Davia J., Cheithin M., Bedynek J.: Sinus venosus atrial septal defect: analysis of 50 cases. Amer. Huart J. 85: 177, 1973

24 Honcoock E.: Coronary sinus rhythm in sinus venosus defect and persistent left superior vena cava. Amer. J. Cardiol. 14: 608, 1964.

25 Hastreiter A.R., Radriguez-Coronat A.: Anomalous inferior cava with azygos continuation, high (sinus venosus) atrial septal defect and alteration of sino atrial thythm. Amer. J. Cardiol, 21: 575, 1968.

26 Crayler G.G.: Spontaneous functional closure of symptomatic atrial septal defect. New Engl. J. Med. 276: 65,1967 .

27 Cumming G.R.: Functional closure of atrial septal defects. Amer, J. Cardiol. 22: 888, 1968.

28 Hartmann A.F., Elliot L.: Spontaneous physiologic closure of an atrial septal defect after infancy. Amer. J. Cardiol, 19: 290, 1967.
${ }^{29}$ Modhu M.R.: Serial Hemodynamic observation in secundum atrial septal defect with special reference to spontaneous closure. Amer. J. Cardiol. 32: 978, 1973.

30 Perloff J.K.: Therapeutic of nature. The invisible suture of spontaneous closure. Amer. Heart J. 82: $581,1971$.

3 I Tinamis G.C., Gordon S., Reed J.O.: Spontaneous closure of an atrial septal defect. J.A.M.A. 196: 137. 1966.

32 Allamand J., Sunkel W., Spoerer A., Castillo P., González $H$., Piwonka G., Vúncos J., Arriagada $M$. Vege E.-. Tratamiento quirúrgico de la CLA. Arch. Soc. Cir. Chile I8 (2): 207, 1966.

33 Salvestrini H., Dubernet J., Valdivieso J., Lucchini A., Moturana $G_{.}$, Allende $M$., Cosanegra $P$., Wilson C. Badia W., Dioz S.: Tratamiento de a CIA con circulación extracorpórea. Arch. Soc. Cir. Chile 18: $202,1966$. 